\title{
Ion-polaron interaction in modified tellurite glasses
}

\begin{abstract}
Glasses formed by different oxides are of uppermost importance because they are developed for many applications in electronics, optics, biology, etc. Oxide glasses are built by a three-dimensional network of corner connected oxygen polyhedral that can be modified by the inclusion of other oxides such as transition metal oxides. In this work, the polaron conductivity due to the presence of transition metal oxides in tellurite glasses is analyzed in systems containing alkaline (AO) and alkaline earth oxides (AEO). It is explained how the presence of metal cations (AO or AEO) affects the displacement of the polaron through the network due to the ion-polaron interaction.
\end{abstract}

Keywords: glasses, oxide creates, polaron, cations, polarization, electron hops, tellurite glasses

\author{
Volume 3 Issue 4 - 2019 \\ Luis A Hernández García, Magalí C Molina, \\ Marianela Zoratti, Evangelina Cardillo, \\ Soledad Terny, Mariela Sola, Marisa A \\ Frechero
}

Departamento de Química, Universidad Nacional del Sur, Argentina

Correspondence: Marisa A Frechero, INQUISURDepartamento de Química, Universidad Nacional del Sur (UNS), Av.Alem 1253, CP 8000, Bahía Blanca, Argentina, Email frechero@uns.edu.ar

Received: June 27, 2019 | Published: July 17, 2019
Abbreviations: AEO, alkaline earth oxides; XRD, X-ray diffraction analysis; $\mathrm{E}_{\mathrm{a}}$, activation energies

\section{Introduction}

Glasses formed by a mix of oxides are interesting for many technological applications. Their physical properties change according to the constituent oxides. Every oxide creates a three-dimensional network built by corner connected oxygen polyhedral, with different coordination number. The oxides can be either glass formers or glass modifiers, granting the final product specific characteristics. Thus, it is of utmost importance which oxides to incorporate to the original mix. In this work, we analyze the polaron conductivity in the presence of large alkaline cation concentrations. We studied oxide glasses formed by $\mathrm{TeO}_{2}$ modified by the incorporation of transition metal oxides: $\mathrm{V}_{2} \mathrm{O}_{5}, \mathrm{Cu}_{2} \mathrm{O}$, and $\mathrm{MoO}_{3}$. Additionally, such tellurite glasses contain $\mathrm{Na}_{2} \mathrm{O}$ or $\mathrm{MgO}$. Holstein ${ }^{1}$ proposed in 1959 for materials with low charge carrier mobility $(<0.1 \mathrm{~cm} / \mathrm{V}$.s $)$ that an electron trapped in the lattice would not be able to move unless the lattice could move together with it. Such proposal gave birth to the polaron concept. The explanation is that the charge carrier (the electron) induces a dipole moment on its neighboring and both move together, i.e. the polaron. Therefore, polaron conductivity involves the displacement of polaron in a material. When the material is a glass the charge carrier and the distortion in its surrounding move through the glassy matrix. For that reason, in this work we study how the presence of alkaline and alkaline earth cations affects the polaron transportation in the tellurite glasses.

\section{Experimental}

The glasses studied in this work have the general formula: $x \mathrm{M}_{\mathrm{i}} \mathrm{O}$ $(1-x)\left[0.5 \mathrm{~V}_{2} \mathrm{O}_{5} 0.5 \mathrm{MoO}_{3}\right] 2 \mathrm{TeO}_{2}(\mathrm{M}=\mathrm{Mg}, \mathrm{Na}, \mathrm{Cu})$ and were prepared by a standard melt quenching technique starting from reagent grade chemicals. Appropriate amounts of the components were well mixed and placed in a platinum crucible and heated at $1073 \mathrm{~K}$ in an electric furnace for half an hour, frequently shaking the crucible to ensure homogenization. The molten material was poured on a preheated aluminum plate forming isolated drops and finally annealed for 2 hours at $450 \mathrm{~K}$. The amorphous character of the samples was tested by X-Ray Diffraction analysis (XRD) with a Bruker D8 Advance diffractometer in continuous scan mode with a copper anode and $45 \mathrm{kV}-30 \mathrm{~mA}$ at room temperature in the $2 \theta$ range: $10^{\circ}-60^{\circ}$. Electrical characterization of the samples Agilent 4284A analyzer within the range of frequencies from $20 \mathrm{~Hz}$ to $1 \mathrm{MHz}$ as a function of temperature always $15 \mathrm{~K}$ below its $T_{g}$ to avoid sample structural changes. Density measurements were done following the Archimedean's method using 2-propyl alcohol as secondary displacement medium.

\section{Results and analysis}

The studied glassy systems are: (Table 1).

Table I The studied glassy systems.

\begin{tabular}{ll}
\hline Glass name & Glass formula \\
\hline MgVMoTe & $x \mathrm{MgO}(\mathrm{I}-x)\left[0.5 \mathrm{~V}_{2} \mathrm{O}_{5} 0.5 \mathrm{MoO}_{3}\right] 2 \mathrm{TeO}_{2}$ \\
CuVMoTe & $x \mathrm{Cu}_{2} \mathrm{O}(\mathrm{I}-x)\left[0.5 \mathrm{~V}_{2} \mathrm{O}_{5} 0.5 \mathrm{MoO}_{3}\right] 2 \mathrm{TeO}_{2}$ \\
NaVMoTe & $x \mathrm{Na}_{2} \mathrm{O}(\mathrm{I}-x)\left[0.5 \mathrm{~V}_{2} \mathrm{O}_{5} 0.5 \mathrm{MoO}_{3}\right] 2 \mathrm{TeO}_{2}$ \\
\hline
\end{tabular}

\section{Theoretical background}

Considering that the polaron hopping theory ${ }^{2}$ was originally used to explain transport in doped or non-doped semiconductors where electrons are distributed in a hydrogen-type orbital according to the wave function: $\varphi=\varphi_{0} \exp (-2 \alpha R)$, the electron is localized according to a landscape of potential values created by the modifier ion incorporated in the glassy matrix. A competition appears between the potential energy variation and the distance that the electron can hop. Therefore, the expression for the electron hopping rate $(\eta)$ to another site at a distance $R$ with carrier energy $\Delta W$ is: $\eta=\eta_{0} \cdot \exp (-2 \alpha R) \cdot \exp (-D W / k T)$ and $\eta$ results proportional to the electrical conductivity $(\sigma)^{2}$. The number of transition metal ions per unit volume in the glassy matrix gives the ion-ion average distance, which influences the electron hop. The factor $\exp (-2 R \alpha)$ in the hopping rate equation is the overlap integral and when $\alpha \sim 0$ it is accepted that the hopping mechanism is adiabatic, and the semiconducting 
behaviour is controlled by the activation energy. When the hopping mechanism is non-adiabatic $(\alpha \neq 0)$ the $\sigma_{\mathrm{dc}}$ is proportional to $\exp (-\mathrm{W} /$ $\mathrm{kT}$ ). Considering that in the polaronic transport theory there are two characteristic lengths: the average distance between sites $(\mathrm{R})$ and the polaron radius $\left(r_{p}\right)$, an estimation of the localized polarization the electron hops from $\mathrm{M}^{\text {red+ }}$ to $\mathrm{M}^{\text {oxid+ }}$ ion depends on both quantities. In our work, we compute $(\mathrm{R})$ and $\left(\mathrm{r}_{\mathrm{p}}\right)$ from the impedance data and we analyse such values as function of the AO and AEO content in our tellurite glasses. Both lengths, $\mathrm{R}$ and $\mathrm{r}_{\mathrm{p}}$, are calculated based on the glass composition and density applying the following equations:

$$
\begin{gathered}
R=N^{-1 / 3} \\
r_{p}=0.5(\pi / 6)^{1 / 3} R
\end{gathered}
$$

Where $\mathrm{N}$ is the number of metal transition ions per molar volume (Table 2). Molar Volume $\left[\mathrm{V}_{\mathrm{M}} \mathrm{cm}^{3}\right], \mathrm{R}[\mathrm{nm}]$ and $\mathrm{W}[\mathrm{eV}]$, values of $\mathrm{W}$ estimated from experimental dc conductivity data (slope in Figure 1).

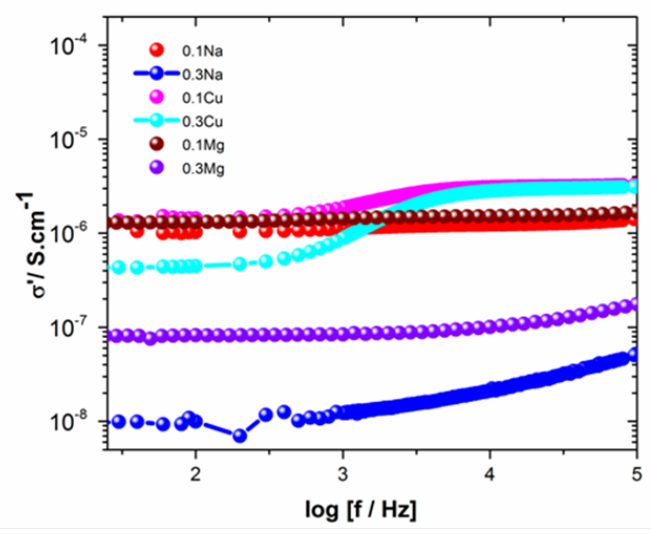

Figure I Real part conductivity spectra at $180^{\circ} \mathrm{C}$.

The tellurite glasses studied here could have two possible electrical carriers: polarons and/or ions according to the content of the $\mathrm{AO}$ or AEO. Considering that vanadium oxide is present in the glassy matrices and they -the vanadium ions- appear in mixed oxidation states, i.e. $\mathrm{V}^{\text {reduced }}$ and $\mathrm{V}^{\text {oxidized }}$, the polaronic conductivity results from an electron transfer between those centers ${ }^{3-6}$. As the electron travels within the matrix a distortion emerges, and they move together as a polaron and the dc conductivity in the system follows the equation:?

$$
\sigma=c \cdot(1-c) \cdot N \cdot \frac{e^{2} R^{2} v_{o}}{6 k T} \cdot \exp [-2 \alpha R] \cdot \exp \left[-\frac{W}{k \cdot T}\right]
$$

Where $\mathrm{c}$ and (1-c) are the occupied and available sites (V $\mathrm{V}^{\text {reduced }}$ and Voxidized); $R$ is the average distance between two adjacent $\mathrm{V} ; v_{\mathrm{o}}$ is the phonon frequency; $\alpha$ is the tunneling factor; $W$ is the activation energy; $\mathrm{k}_{\mathrm{B}}$ and $\mathrm{T}$ are the Boltzmann constant and the absolute temperature. Consequently, the conductivity temperature dependence is (Figure 2):

$$
\sigma \cdot T=\sigma_{o} . \exp \left[-\frac{W}{k \cdot T}\right]
$$

The $\mathrm{W}$ in Eq. 4 is the potential barrier that the electron has to overcome due to the phonon vibrations between the $V^{\text {red }}$ and the Voxid. ${ }^{8}$ From the results we learn that as the vanadium ions content diminishes, such potential barrier increases because of the increase in R. ${ }^{9,10}$ Therefore, the conductivity decreases. Previously it was demonstrated that only the vanadium ions are engaged in the process because the incorporation of molybdenum ions does not involve a higher energy barrier. ${ }^{11}$ As the $\mathrm{V}_{2} \mathrm{O}_{5}$ concentration decreases also the ratio $\mathrm{V}^{\text {red }} / \mathrm{V}^{\text {oxid }}$ is conditioned because it depends strongly on its redox surroundings. ${ }^{12,13}$ Figure 3 shows a conductivity diminution as the $\mathrm{V}_{2} \mathrm{O}_{5}$ content is replaced by $\mathrm{MgO}$ or $\mathrm{Na}_{2} \mathrm{O}$; but almost any change is observed when $\mathrm{Cu}_{2} \mathrm{O}$ replaces $\mathrm{V}_{2} \mathrm{O}_{5}$. Even so, the reduction in the content of $\mathrm{V}_{2} \mathrm{O}_{5}$ causes a similar change on the $\mathrm{V}^{\text {red }}$ - $\mathrm{V}^{\text {roxid }}$ average distance as we can see from $\mathrm{R}$ values in Table 2, but the replacement of $\mathrm{V}_{2} \mathrm{O}_{5}$ by $\mathrm{Cu}_{2} \mathrm{O}$ does not change the conductivity, it remains almost constant. Therefore, it is possible to assume that the polaron hopping is not affected by the mixed type of sites (weather it was $\mathrm{V}_{2} \mathrm{O}_{5}$ or $\mathrm{Cu}_{2} \mathrm{O}$ ) and a unique path of reduced/oxidized sites is present for the polaron hopping (Figure 3). Further, it is interesting to notice that when $\mathrm{MgO}$ replaces $\mathrm{V}_{2} \mathrm{O}_{5}$ the conductivity diminishes because of the diminution of polaron concentration but also because of the potential landscape change due to the incorporation of the $\mathrm{MgO}$ in the glassy matrix. In addition, this change is stronger when $\mathrm{Na}_{2} \mathrm{O}$ is incorporated because the $V^{\text {red }} \rightarrow V^{\text {oxid }}$ path is severely interrupted and the ionic conductivity due to free mobile $\mathrm{Na}^{+}$also disturbs the polaron transport because the ion-polaron interaction appears. Therefore, the mixed conductivity explains the changes of the $\mathrm{W}$ values showed in Table 2. In 1996 Bazan et al., ${ }^{14}$ confirmed the Kraevsky's findings ${ }^{15}$ and explained the hypothesis of the interactions between polarons and mobile cations. Such interaction is the source of the anomaly observed in the conductivity behavior because the polarons are attracted by the opposite charge of the mobile cations. Then, a neutral pair moves, and it does not contribute to the electrical conductivity. The authors in that work explained the ion-polaron effect, the maxima in the activation energies $\left(\mathrm{E}_{\mathrm{a}}\right)$ and the pre-exponential factors of the conductivity Arrhenius expression $\left(\sigma_{0}\right)$. The ion-polaron interaction causes the reduction of the electrical conductivity in mixed conductors due to the slower charges displacements caused by the Coulomb interactions. Such explanation allows for better understanding of the relationship between polaronic and ionic in the whole glass conductivity. (Figure 4).

Table 2 Molar Volume $\left[\mathrm{V}_{\mathrm{M}} \mathrm{cm}^{3}\right], \mathrm{R}[\mathrm{nm}]$ and $\mathrm{W}[\mathrm{eV}]$, values of $\mathrm{W}$ estimated from experimental dc conductivity data (slope in Figure 2).

\begin{tabular}{llllllllll}
\hline & \multicolumn{3}{c}{$\mathbf{V}_{\mathbf{M}}\left[\mathbf{c m}^{3}\right]$} & \multicolumn{3}{c}{$\mathbf{R}[\mathbf{n m}]$} & \multicolumn{3}{c}{$\mathbf{W}[\mathbf{e V}]$} \\
\hline $\mathrm{x}$ & $\mathrm{Mg}$ & $\mathrm{Cu}$ & $\mathrm{Na}$ & $\mathrm{Mg}$ & $\mathrm{Cu}$ & $\mathrm{Na}$ & $\mathrm{Mg}$ & $\mathrm{Cu}$ & $\mathrm{Na}$ \\
0.1 & 22.90 & 33.86 & 33.79 & 0.50 & 0.57 & 0.57 & 0.52 & 0.47 & 0.49 \\
0.2 & 22.87 & 33.03 & 32.60 & 0.52 & 0.59 & 0.59 & 0.54 & 0.48 & 0.49 \\
0.3 & 22.80 & 32.06 & 32.88 & 0.55 & 0.61 & 0.61 & 0.55 & 0.53 & 0.64 \\
0.4 & 23.43 & 31.46 & 31.27 & 0.58 & 0.64 & 0.64 & 0.61 & 0.57 & 0.72 \\
0.5 & 23.62 & 30.96 & 30.77 & 0.62 & 0.68 & 0.67 & 0.64 & 0.62 & 1.03 \\
\hline
\end{tabular}



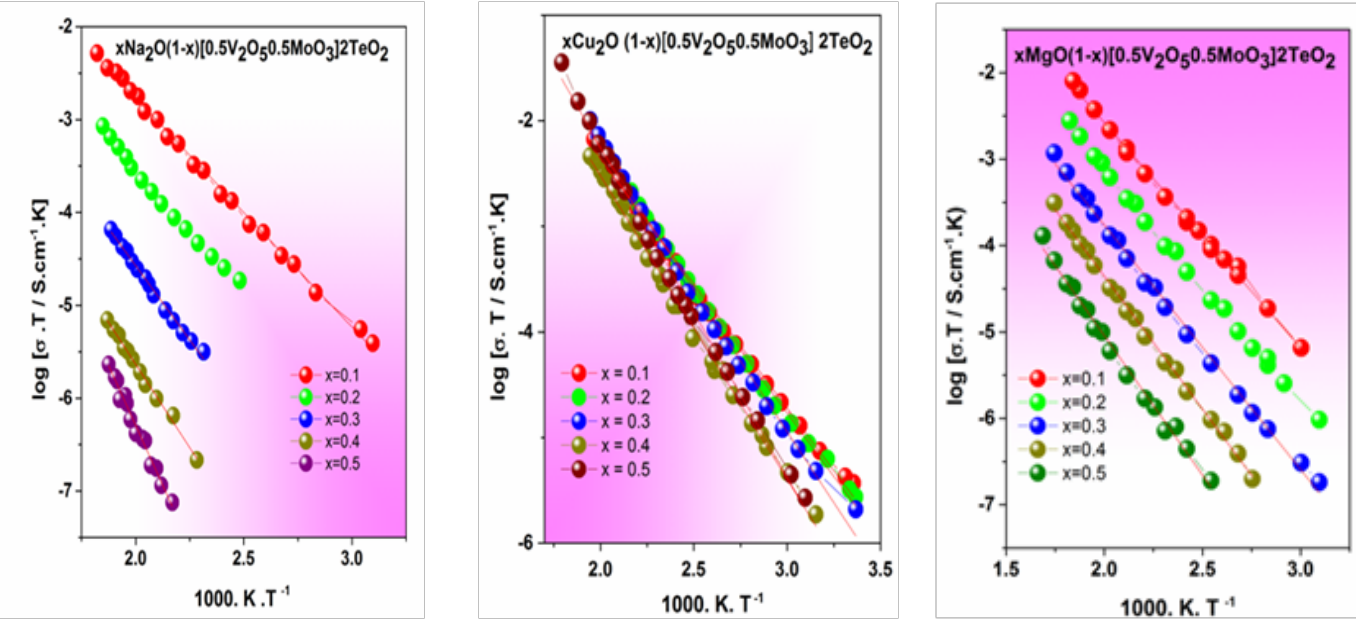

Figure 2 Temperature dependence of glasses dc conductivity: $x \mathrm{Na}_{2} \mathrm{O}(\mathrm{I}-x)\left(0.5 \mathrm{~V}_{2} \mathrm{O}_{5} 0.5 \mathrm{MoO}_{3}\right) 2 \mathrm{TeO}_{2} ; x \mathrm{Cu}_{2} \mathrm{O}(\mathrm{I}-x)\left(0.5 \mathrm{~V}_{2} \mathrm{O}_{5} 0.5 \mathrm{MoO}_{3}\right) 2 \mathrm{TeO}_{2}$ and $x \mathrm{MgO}(\mathrm{I}-x)$ $\left(0.5 \mathrm{~V}_{2} \mathrm{O}_{5} 0.5 \mathrm{MoO}_{3}\right) 2 \mathrm{TeO}_{2}$

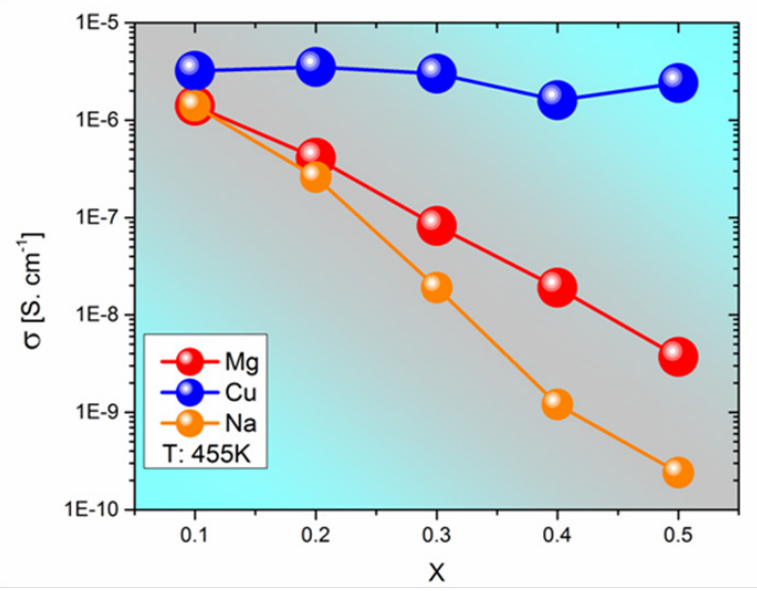

Figure 3 Isotherm of conductivity at around $180^{\circ} \mathrm{C}$ as a function of modifier oxide content $(x)$ of systems: $x M g O(I-x)\left(0.5 \mathrm{~V}_{2} \mathrm{O}_{5} 0.5 \mathrm{MoO}_{3}\right) 2 \mathrm{TeO}_{2} ; \mathrm{xCu}_{2} \mathrm{O}(\mathrm{I}-\mathrm{x})$ $\left(0.5 \mathrm{~V}_{2} \mathrm{O}_{5} 0.5 \mathrm{MoO}_{3}\right) 2 \mathrm{TeO}_{2}$ and $x \mathrm{Na}_{2} \mathrm{O}(\mathrm{I}-\mathrm{x})\left(0.5 \mathrm{~V}_{2} \mathrm{O}_{5} 0.5 \mathrm{MoO}_{3}\right) 2 \mathrm{TeO}_{2}$.

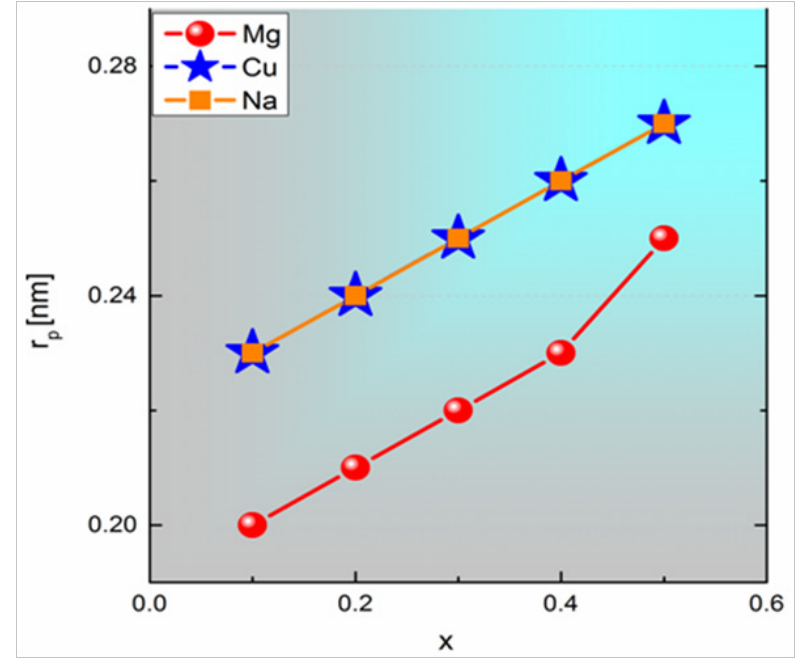

Figure 4 Temperature dependence of dc conductivity of systems: $x M g O(I-x)\left(0.5 \mathrm{~V}_{2} \mathrm{O}_{5} 0.5 \mathrm{MoO}_{3}\right) 2 \mathrm{TeO}_{2}$. 
The electrical conductivity results plotted in Figure 3 can be understood through the pre-exponential factor of the Eq. 3. In such equation, the displacement of one electron from site to site between two sites with similar energy level is considered, the phonon scattering gives the equalization of two adjacent positions and the overlapping of the wave function is the factor $\exp (-2 \alpha \mathrm{R})$. Therefore, in the following equation: ${ }^{16}$

$$
\sigma_{o}=c .(1-c) \cdot N \cdot \frac{e^{2} R^{2} v_{o}}{6 k T} \cdot \exp [-2 \alpha R]
$$

It is assumed that there are three fundamental factors: $1-[c$. (1c)] is the probability of having a donor and an acceptor site in an adjacent position; 2- $\left[v_{o} \exp \left(W_{e}+W_{D} / 2 k T\right)\right]$ : is the probability of two adjacent sites having the equivalent energy; and $3-[\exp (-2 \alpha R)]$ is the probability of an electron to hop from site to site, i.e the tunneling factor. Therefore, from the conductivity results in Figure 3 \& Figure 2 and the parameters in the Table 2 above, it is

easy to understand computing

$$
\frac{\sigma_{0 M g}}{\sigma_{0 N a}} \approx\left\{\left(\ln N_{M g}+2 \ln R_{M g}-2 \alpha_{M g} R_{M g}\right)-\left(\ln N_{N a}+2 \ln R_{N a}-2 \alpha_{N a} R_{N a}\right)\right\}
$$

, and in analogous manner for $\sigma_{0 \mathrm{Cu}} / \sigma_{0 \mathrm{Mg}}$. Considering that:

$$
\begin{aligned}
& {\left[-2 \alpha_{M g} R_{M g}+2 \alpha_{N a} R_{N a}\right] \cong 0} \\
& {\left[2 \ln R_{M g}-2 \ln R_{N a}\right]<0} \\
& \left.\left.\left[\ln N_{M g}-\ln N_{N a}\right] \Rightarrow\right\rangle\right\rangle 1
\end{aligned}
$$

We conclude: $N_{C u}>N_{M g}$ and similarly that $N_{C u}>N_{M g}$.

This is notorious result. Such result is in good agreement with the Anderson theory (1958) which considered that a random small nonperiodic potential affected the migration of an electron in a system. ${ }^{10}$

\section{Conclusion}

The analysis made here reveals that the entity originated from the interaction between a charge carrier (electron) and the dipole moment induced on surrounding, so called polaron, causes the main conductivity phenomenon in a material. When the material is a tellurite glass, the charge carrier and its distortion in its surrounding are strongly affected by the presence of free mobile ions in the glassy matrix as Na-system and $\mathrm{Cu}$-system show. Therefore, the presence of mobile cations affects the polaron through the change on the landscape and the model condition of the original theory developed by Anderson is not strictly satisfied. The existence of a not small perturbation given by mobile cations involves a strong effect on the non-periodic potential added to the formation of ion-polaron neutral entity in the studied modified tellurite glassy matrices.

\section{Acknowledgements}

I. L.A.H.G. and M.Z. are fellows of CONICET Argentina.

II. M.C. M. is fellow of ANPCyT Argentina

III. E. C. C. is Researcher Fellow of CIC PBA Argentina.

IV. S.T and M.A.F are Researcher Fellow of CONICET Argentina.

\section{Funding details}

This work has been possible to the financing support by Universidad Nacional del Sur (PGI 24/Q078) and PICT 2016-0101

\section{Conflict of interest}

Authors declare that there is no conflict of interest.

\section{References}

1. Holstein T. Studies of polaron motion: Part II. The "small" polaron. Ann Phys. 1959;8:343-389.

2. Banerjee A, Pal S, Rozenberg E, et al. Adiabatic and non-adiabatic smallpolaron hopping conduction in $\mathrm{La}_{1-} \mathrm{xPb}_{\mathrm{x}} \mathrm{MnO}_{3+\delta}(0.0 \leq \mathrm{x} \leq 0.5)$-type oxides above the metal-semiconductor transition. J Phys Condens Matter. 2001;13:9489-9504.

3. Austin IG, Mott NF. Polarons in crystalline and non-crystalline materials. Adv Phys.1969;18:41-102.

4. Vijaya Prakash G, Narayana Rao D, Bhatnaga AK. Linear optical properties of niobium-based tellurite glasses. Solid State Commun. 2001;119:39-44.

5. Szu S, Shing Gwo Lu. Phys B. AC impedance studies of $\mathrm{V}_{2} \mathrm{O}_{5}$ containing glasses. 2007;391:231-237.

6. PE di Prátula, Terny S, Cardillo EC, et al. The influence of transition metal oxides type $\mathrm{M}+\mathrm{M}++$ on the vanadium-tellurite glasses electrical behavior. Solid State Sciences. 2015;49:83-89.

7. Ghosh J. Transport mechanism in semiconducting glassy silicon vanadates. Appl Phys. 1993;74:3961-3965.

8. Murawski L, Chung C, Mackenzie J. Electrical properties of semiconducting oxide glasses. J Non Cryst Solids. 1979;32:91-104.2

9. Terny S, PE di Prátula, De Frutos J, et al. Dielectric relaxation of vanadiummolybdenum tellurite glasses modified by alkaline-earth oxides. Journal of Non-Crystalline Solids. 2016;444:49-54.

10. Terny s, De la Rubia MA, De Frutos J, et al. A new transition metaltellurite glass family: Electrical and structural properties. Journal of NonCrystalline Solids. 2016;433:68-74.

11. Frechero MA, Quinzani O, Pettigrosso R, et al. IR absorption spectra of lithium and silver vanadium-tellurite based glasses. J Non Cryst Solids. 2007;353:2919-2925.

12. Das BB, Mohanty D. Activation energies of hopping of vanadium $3 \mathrm{~d} 1$ small polaron in $0.7 \mathrm{~V}_{2} \mathrm{O}_{5}-(0.3-\mathrm{x}) \mathrm{P}_{2} \mathrm{O}_{5}-\mathrm{x}\left(\mathrm{As}_{2} \mathrm{O}_{3} / \mathrm{Sb}_{2} \mathrm{O}_{3}\right)(\mathrm{x}=0,0.05)$ glasses by EPR spectroscopy and DC electrical conductivity measurements. Indian J Chem 2006;45:2400-2405.

13. Sen S, Ghosh A. Electrical properties of semiconducting barium vanadate glasses. J Appl Phys. 2000;387(7):3355.

14. Bazan JC, Duffy JA, Ingramb MD, et al. Conductivity anomalies in tungstate-phosphate glasses: evidence for an ion-polaron interaction? Solid State Ionics. 1996;86-88:497-501.

15. Kraevskii SL, Solinov VF. Hierarchical Percolation and Electrical Conduction of Glasses in the $50 \mathrm{WO}_{3}-(50-\mathrm{x}) \mathrm{P}_{2} \mathrm{O}_{5}-\mathrm{xNa}_{2} \mathrm{O}$ System. Glass Physics and Chemistry. 2006;32(6):629-633.

16. Lebrun N, Levy M, Souquet JL. Eectronic conductivity in glasses of the $\mathrm{TeO}_{2}-\mathrm{V}_{2} \mathrm{Os}-\mathrm{MoO}_{3}$ System. Solid State Ionics. 1990;40/41:718-722. 